\title{
Diversification in Urban Functions as a Measure of Metropolitan Complexity
}

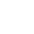

\section{Abstract}

Newly emerging relationships between form and functions reveal the increasingly complex nature of metropolitan regions. The present study investigates spatial diversification in settlement forms and socioeconomic functions in metropolitan Attica (the administrative region including Athens, the capital of Greece), with the aim to implement a holistic framework assessing urban complexity in contemporary cities. Taken as key components of urban complexity, morphological and functional diversity have been analyzed using multi-domain indicators that describe settlement characteristics (land-use, soil sealing, building use, vertical profile of buildings, building age, construction materials) and socioeconomic functions (economic base, working classes, education levels, population age structure, composition of non-native population by citizenship, distribution of personal incomes), thus providing a comprehensive description of local-scale diversification in urban structures. A correlation analysis was used to verify the spatial coherency between individual dimensions of urban diversification. Analysis of global and local Moran's spatial autocorrelation indexes reveals specific gradients of urban diversification that discriminate morphological attributes from socioeconomic functions. Municipalities were profiled on the base of Pielou's evenness indexes for each urban dimension: a factor analysis indicates latent patterns characterizing areas with high and low diversification in metropolitan functions. Urban and rural municipalities were respectively characterized as the most and less diversified in the study area, with peri-urban municipalities ranking in-between, evidencing a diversification gradient correlated with the distance from downtown Athens. A multidimensional analysis of the most relevant dimensions of metropolitan complexity proved to be a promising tool for monitoring urban gradients, polycentric development and (latent) socioeconomic transformations in contemporary cities.

Key words: Social mix, Economic structure, Spatial inequalities, Evenness, Mediterranean city.

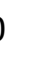

\section{Introduction}


1 With metropolitan growth progressively more oriented toward residential decentralization, spatial rebalancing and functional polycentrism, an intense debate on the emerging relationships between urban forms and functions has consolidated in recent years (Markusen and Schrock, 2006; Burger and Meijers, 2012; Hirt, 2012; Kroll and Kabisch, 2012). While cities constitute an unsurpassed engine of growth for neighbouring regions, urban development follows distinctive paths reflecting sometimes place-specific transformations (Meijers and Burger, 2010). diversity in forms and functions is intimately related with metropolitan development, where diverse and internallydiversified cities develop and consolidate (Talen, 2006).

Socioeconomic functions are generally oriented along the urban gradient, as inner cities and the surrounding urban centres concentrate most of activities, promoting a higher diversification of metropolitan structures (Parr, 2004; O'Donoghue and Townshend, 2005; Zambon et al., 2018). Spatial features of urban expansion, such as infilling, edge-expansion or leapfrogging, have been extensively studied in literature to identify specific drivers (topological, socioeconomic, or related to place-specific factors such as accessibility) and consequences in terms of compactness or sprawl of the resulting urban forms (Mustafa et al., 2018). This spatial pattern may have different declinations in specific contexts: urban districts with diverging ranks in the urban hierarchy may assume a comparable structure and, conversely, areas with similar size and population density may have divergent structures, hosting a different composition of economic activities and social functions (O'Donoghue, 1999). Moreover, functional changes in metropolitan hierarchies occur through complex trajectories of growth, since urban expansion no longer occurs through physical addition of peripheral development units at the most basic scale, stimulating a progressively more polycentric and decentralized growth (Duranton and Puga, 2000; Ottaviano and Peri, 2006; Zambon et al., 2017).

In these regards, settlement expansion around sub-centres have altered metropolitan structures traditionally oriented along urban gradients, influencing the spatial dimension of urban diversification (Turok and Bailey, 2004; Parr, 2008; Pili et al., 2017). Exurban development has demonstrated to create a sort of 'metropolitan continuum', with medium-density settlements embedded into a fragmented landscape, with spatially-varying implications for processes of change at the base of urban complexity (Torrens, 2008; Vasanen, 2012; Salvati, 2013, 2014; Serra et al., 2014; Kasanko et al., 2006; Hahs and McDonnell, 2006; Serra et al., 2014; Salvati and Carlucci, 2016; Di Feliciantonio and Salvati, 2015). With changes in the spatial organization of metropolitan regions driven by dispersed urbanization, the analysis of metropolitan continuums based on 
1 diversification indexes is a challenging task in regional science, planning and geography 2 (Ellerman, 2005; Dahly and Adair, 2007; Munafò et al., 2013; Salvati et al., 2016a). By identifying 3 socioeconomic transformations along urban gradients, approaches based on a multidimensional analysis of diversification in key metropolitan functions demonstrated suitable to distinguish inner cores and suburban areas, providing policy-oriented classifications of urban and rural districts (Colantoni et al., 2015; Comer and Greene, 2015; Cuadrado-Ciuraneta et al., 2017; Duvernoy et al., 2018).

Contributing to the inherent complexity of contemporary cities, functional diversification and urban entropy are taken as relevant traits of metropolitan growth in Europe (Batty and Longley, 1994; Page et al., 2001; Cabral et al., 2013; Kazemzadeh-Zow et al., 2017). On average, European cities reveal a greater mix and integration of socioeconomic functions than Northern American towns (e.g. Carlucci et al., 2017). Particularly European Mediterranean cities are examples of urban diversification and spatial entropy in their constituent elements, sharing common characters while reflecting distinctive history, socioeconomic development and settlement models (Beriatos and Gospodini, 2004; Grekousis et al., 2013; Di Feliciantonio and Salvati, 2015; Zitti et al., 2015). Among the main forces shaping urban transformations in Mediterranean Europe, economic polarization and social segregation became particularly relevant in metropolitan expansion and regional disparities (Malheiros, 2002; Maloutas, 2007; De Rosa and Salvati, 2016; Rontos et al., 2016). Additionally, Mediterranean cities have often expanded in a partly unregulated manner resulting in disordered urban mosaics that reflect a fragmented economic base (Maloutas, 1993; Stanley, 2012; Colantoni et al., 2016; Salvati, 2016). Long-term development processes have frequently reflected heterogeneous and self-organized metropolitan systems characterized by economic disparities, social isolation, settlement scattering, and a fractal morphology (Carlucci et al., 2017). By this way, Mediterranean cities have represented key examples of socioeconomic transformations leading to morphologically-dispersed and functionally-discontinuous cities (Pili et al., 2017).

By contributing to a better comprehension of urban complexity, the present work implements an original framework to the analysis of complex metropolitan systems based on the use of multidomain indicators and spatial statistics. The proposed approach sheds light on the spatial pattern of diversification in multiple urban functions and morphological attributes illustrating economic, social and environmental aspects at both local and regional scale in the Athens' metropolitan region (Greece). Athens is considered a representative case of Mediterranean cities moving from a 
1 mostly informal expansion to planned development toward urban sustainability and a more

2 balanced spatial structure. The complex (and, for some aspects, still unclear) relationship between

3 socioeconomic functions and urban morphology in Athens, as in many other Mediterranean cities

4 (Di Feliciantonio and Salvati, 2015), justifies a multivariate analysis of urban diversification in such

5 territorial context, under the hypothesis that metropolitan complexity reflects the increasing

6 diversification in economic, environmental and social dimensions along urban gradients.

7

\section{Methodology}

\subsection{Study area}

The Athens Metropolitan Region (AMR) mostly coincided with the boundaries of the administrative region of Attica, Greece. Up to 2011, the AMR was divided in 4 prefectures (Athens, Piraeus, East Attica and West Attica) and administered by 114 municipalities extending 3,000 km², with population density $>1200$ inhabitants $/ \mathrm{km}^{2}$ (2011). With the so called 'Kallikratis' law, a new local administrative regime was adopted in Greece since 2012, reducing the number of municipalities in the study area to nearly 60. Since statistical data referring to 2010 or 2011 were used as the main information source, the spatial structure adopted in this study refers to the administrative division (the so called 'Kapodistrias' structure) in law at that time. The AMR includes dense urban and hyper-compact districts such as downtown Athens and Piraeus, suburban municipalities bordering the strictly urban area and rural municipalities with moderately-low accessibility and a marginal role in the regional economic system. The urban core is traditionally associated with the 'Greater Athens area', a district with compact urban fabric extending $430 \mathrm{~km}^{2}$ and administered by 58 municipalities ('Kapodistrias' structure), hosting most of the population (85\% in 2011) and the economic activities of the AMR.

\subsection{Logical framework}

Taken as basic components of metropolitan complexity, 12 dimensions of urban diversification were considered including morphological aspects (land-use, soil sealing, building use, vertical profile of buildings, building age, construction materials) and socioeconomic functions (economic base, working classes, education levels, population age structure, composition of non-native 
1 population by citizenship, distribution of personal incomes). To allow a coherent analysis of localscale patterns of urban diversification, municipalities were adopted as the elementary analysis' domain in this study. Being easily derived from statistical data sources at the municipal scale, such dimensions are intended to provide a comprehensive description of metropolitan complexity in the Mediterranean region and, possibly, in other socioeconomic contexts worldwide.

\subsubsection{Morphological attributes}

Six variables organized in different classes were computed at the spatial scale of municipalities: 1) land-use (hereafter 'land'); 2) soil sealing profile ('soil'); 3) building use ('use'); 4) vertical profile of buildings ('vert'); 5) building age ('buil'); 6) construction materials ('mat'). Landscape composition (percent class area in total municipal area) was derived from a high-resolution land-use map (1: 10,000 scale) referring to 2012 and disseminated through the European Urban Atlas (UA) initiative on behalf of the Global Monitoring for Environment and Security (GMES) framework adopting a nomenclature system composed of 20 classes (built-up areas: code 1, cropland: code 2, and forests: code 3).

A soil sealing profile for each municipality was derived from a $100 \mathrm{~m}$-grid land imperviousness map referring to 2012 and disseminated by Land Copernicus initiative in collaboration with the European Environment Agency (2011). This considers all pavement structures (roads, sidewalks, driveways and parking lots) that are covered by impenetrable materials such as asphalt, concrete, brick, stone and rooftops with continuous degree of land imperviousness ranging from $0 \%$ to $100 \%$. Classification accuracy of built-up and non-built-up areas was higher than $85 \%$ per hectare, with omission and commission errors kept below 15\% (European Environment Agency, 2011). A soil sealing profile for each municipality of the study area was determined by computing percent area in total municipal area of 22 classes with different land imperviousness intensity $(0 \%, 1-5 \%, 6$ $10 \%, \ldots, 91-95 \%, 96-99 \%, 100 \%)$. For both land-use and soil sealing variables, calculations were implemented using the 'Tabulate areas' tool provided with ArcGIS software (ESRI Inc., Redwoods, USA) after the overlap between the land imperviousness map and a municipal boundary shapefile.

The other 4 variables assessing settlement characteristics were derived from the national census of buildings carried out by Greek Statistical Authority (ELSTAT) aggregating elementary data (2011) at municipal scale. Building types were classified using 18 categories distinguishing residential from industrial, commercial and service use and the percent share of each building class in total 
1 municipal building stock was calculated accordingly. Building age was determined using 10 classes assessing construction time period and computing the percent share of buildings by age class in total building stock. Vertical profile of buildings was assessed considering 6 classes that distinguish buildings according to their number of floors and calculating the percent share of buildings by height class in total building stock. Finally, buildings were classified according to the dominant construction material using 6 material types and calculating the percent share of buildings by construction material in total building stock.

\subsubsection{Socioeconomic functions}

Six variables organized in different classes were computed at municipal scale: 1) economic base (hereafter 'econ'); 2) working class composition ('work'); 3) educational level of active population ('educ'); 4) population age structure ('pop'); 5) composition of non-native resident population by citizenship ('fore'); 6) distribution of personal incomes ('inc'). The economic base was assessed considering the number of enterprises in the national business register (referring to 2010) based on a classification of activities composed of 15 sectors and compatible with the NACE-Rev2 nomenclature, calculating the percent share of enterprises by sector in total registered enterprises at municipal scale. Working class composition was studied referring to a classification of workers composed of 24 categories that distinguish different professional positions according to the national census of population and households held in 2011 in Greece. Based on these data, percent share of workers by professional position in total workers was calculated for each municipality of the study area. Education of active population was assessed referring to a nomenclature system with 13 education levels adopted by Greek population census (2011); percent share of active population by education level in total active population was calculated for each municipality. Population structure was investigated using census data that aggregate resident population into 7 age classes and computing percent share of population by age class in total resident population. Composition of non-native population by citizenship was analyzed aggregating resident population in 7 classes (including the most frequent nationalities in the study area and a residual class) based on census data (2011) and computing the percent share of each class in total resident population. Finally, distribution of personal incomes across population was investigated considering elementary data from individual tax declarations (provided by the Hellenic Ministry of Finance and referring to 2014), aggregating resident inhabitants into 10 income classes. 


\subsection{Indexes of urban diversification}

3

Twelve indicators of urban diversification, one for each variable described in section 2.2, were calculated using Pielou's evenness index (J). This index has been extensively used to identify diversity-based functions, providing a comprehensive reading of pertinent aspects of urban growth and change (see references in Zambon et al., 2017). Based on a Shannon diversity function (the so called $\mathrm{H}^{\prime}$ Shannon index grounded on information theory), Pielou's J index is an entropy index standardized to the level of diversification in a given spatial domain (Salvati et al., 2016a), quantifying local-scale diversity and heterogeneity for each variable selected in this study. Ranging from 0 (complete homogeneity) to 1 (the highest heterogeneity according to the level of local-scale diversification), Pielou's J index was calculated as follows:

$$
\mathrm{J}=\mathrm{H}^{\prime} / \mathrm{H}_{\max }
$$

where $\mathrm{H}^{\prime}$ is the Shannon diversity index calculated as:

$$
\mathrm{H}^{\prime}=\sum_{\mathrm{i}=1^{\mathrm{n}}} \mathrm{p}_{\mathrm{i}}^{*} \ln \left(\mathrm{p}_{\mathrm{i}}\right)
$$

where $\mathrm{p}_{\mathrm{i}}$ is the proportion of observations falling in the $i$-th class on the total number of observations for each urban dimension. $\mathrm{H}_{\max }$ is the logarithm of the number of classes with at least one observation.

\subsection{Statistical analysis}

A multi-step approach was implemented in this study with the following objectives: (i) to assess the spatial structure of each dimension of urban diversification, (ii) to identify pair-wise relationships between dimensions of urban diversification, (iii) to characterize the latent interplay between morphological and functional dimensions, (iv) to cluster municipalities on the base of their diversification profile and, finally, (v) to relate the identified diversification profiles to contextual attributes assessing the metropolitan gradient. These objectives were addressed using an exploratory approach integrating spatial statistics (global and local Moran's I spatial autocorrelation indexes) with descriptive analysis/mapping (objective i), parametric and nonparametric correlations (objective ii), a principal component analysis outlining latent relationships among variables (objective iii), hierarchical clustering (objective iv) and a final correlation analysis 
1 (objective v) resuming the latent relationship between local-scale diversification in urban functions

\subsubsection{Spatial autocorrelation analysis}

Global and local Moran's Indexes have proven to be a powerful tool in urban studies (see, among others, Tsai, 2005; Riguelle et al., 2007; Thomas et al., 2012; Salvati et al., 2016b).

Thus, a global Moran's spatial autocorrelation index (z-score) computed on 8 bandwidths (from 5 $\mathrm{km}$ to $40 \mathrm{~km}$ ) was run separately for each dimension of urban diversification using municipalities as the elementary analysis' domain. Local Moran's coefficients of spatial autocorrelation were also calculated and tested for significance. The Moran's scatterplot was used to classify municipalities in the HH (High-High), LL (Low-Low), HL (High-Low) or LH (Low-High) type of spatial autocorrelation regime. HH and LL regimes indicate spatial clustering while HL and LH regimes indicate local heterogeneity associated to a spatial divide reflecting a (more or less steep) gradient in the studied variable.

\subsubsection{Inferential analysis}

A pair-wise correlation analysis using both parametric (Pearson linear product moment) and nonparametric (Spearman rank) coefficients was used to verify the spatial coherency between individual dimensions of urban diversification, testing for significance at $p<0.05$ after Bonferroni's correlation for multiple correlations. A comparison of parametric and non-parametric techniques based on the absolute difference between correlation coefficients (i) may indicate linear (or more complex) forms of the relationship between the studied variables and (ii) allows a coherent analysis of variables displaying a relevant deviation from normality. Coherency in sign and intensity of both parametric and non-parametric coefficients reflects a linear correlation between dimensions of urban diversification. Divergence in sign and intensity of correlation coefficients indicates more complex relationships among urban dimensions.

\subsubsection{Multivariate exploratory analysis}


1 A Principal Component Analysis was run on a data matrix composed of 12 columns (Pielou's J 2 index for each urban dimension) and 114 rows (municipalities) with the aim to extract few

3 (independent) components of urban diversification and to identify the related spatial patterns. A 4 hierarchical clustering using Euclidean distances and Ward's agglomeration rule was carried out 5 separately on urban dimensions and municipalities with the aim to visualize similarities in the 6 spatial distribution of individual components of urban diversification and homogeneous groups of municipalities based on spatially-consistent patterns of urban diversification. A non-parametric

8 (Spearman) correlation analysis was finally run to identify pair-wise relationships between individual dimensions of urban diversification and contextual indicators (density, elevation, proximity to the sea, distance from 4 urban centres - Athens, Piraeus, Maroussi, Markopoulo Messoghias - and municipal surface area), testing for significance at $p<0.05$ after Bonferroni's correction for multiple comparisons.

\section{Results}

\subsection{Assessing dimensions of urban diversification}

The statistical distribution of individual Pielou's J evenness indexes was presented in Table 1. On average, the highest evenness indexes $(>0.7)$ were found for economic structure, working class structure, educational levels, vertical profile of buildings, building construction age, population age structure and personal income distribution. The central district corresponding with the Greater Athens' area emerged for a high diversification in specific morphological attributes (land-use, soil sealing profile, vertical profile of buildings and, to a lesser extent, building use) and socioeconomic dimensions (composition of non-native population by citizenship, distribution of personal income). A reverse diversification pattern was observed for specific morphological dimensions (building age, construction materials) and socioeconomic functions (educational levels). Heterogeneous spatial patterns - with no specific divides between urban and rural districts - were observed for diversification in economic structure, working class composition and population age structure, evidencing few sub-centres with medium-high socioeconomic diversification. 
1 Using 8 bandwidths ranging from $5 \mathrm{~km}$ to $40 \mathrm{~km}$, global Moran's coefficients of spatial autocorrelation showed inherent differences for the dimensions considered in this study (Table 2). Spatially-correlated structures (significance tested at $p<0.05$ ) were observed at all bandwidths for 6 out of 12 dimensions ( 5 morphological attributes and 1 socioeconomic function). Two dimensions (building age and distribution of personal incomes) had a spatially-correlated structure for 7 out of 8 bandwidths. Two dimensions (diversification in working class composition and in educational levels) were spatially-correlated at bandwidths $>15 \mathrm{~km}$. Finally, two dimensions (diversification in economic base and in population age structure) have no significant autocorrelation coefficients, indicating spatial heterogeneity with no specific patterns either at local and regional scale. Local Moran's coefficients of spatial autocorrelation for each municipality of the study area (SM.Figure 1) evidence similar spatial clusters for the following dimensions of urban diversification: land-use, building use, economic base, educational levels, composition of nonnative population by citizenship and distribution of personal income. Municipalities in northeastern Athens' fringe belong to a high-low cluster indicating a significant gradient dividing urban and rural districts. Municipalities classified as high-high clusters were concentrated in both central areas and peri-urban districts for 5 dimensions (population age structure, building age, vertical profile of buildings, soil sealing profile and land-use composition), indicating a spatial structure more oriented toward a metropolitan continuum.

\subsection{Correlation analysis}

Results of a pair-wise correlation analysis based on parametric (Pearson) and non-parametric (Spearman) coefficients are illustrated in Table 3. Significant pair-wise coefficients mainly reflect linear relationships among urban dimensions (similar correlation coefficients for both Pearson and Spearman analysis); more specifically, diversification in land-use and soil sealing profiles have similar correlation profiles, being negatively associated with diversification in building materials (and in population age structure, only for soil sealing profile) and positively associated with diversification in (i) vertical use of buildings, (ii) distribution of personal incomes and (iii) composition of non-native population by citizenship. Diversification of local-scale economic base was correlated positively with working class composition and building age, possibly indicating a higher economic diversification in consolidated urban settlements than in more recently developed districts. Diversity in working class composition increased linearly with diversity in personal 
1 incomes. Diversification in educational levels was correlated positively with diversification in

2 building age and in personal income distribution within the population residing in each

3 municipality. Diversification in buildings' vertical profile was negatively correlated with

4 diversification in building materials and positively correlated with diversification in personal

5 income distribution and in the composition of non-native population by citizenship. Finally,

6 diversification in building materials was negatively correlated with diversification in the composition of personal incomes and non-native population by citizenship.

For some significant pair-wise correlations, the absolute difference between Pearson and Spearman coefficients was particularly high, evidencing a non-linear relationship between (i) educational levels and working class composition and (ii) distribution of personal income and diversification in construction materials. A non-linear correlation was also observed between diversification in the composition of non-native population by citizenship and working class composition (a significant Spearman coefficient together with a non-significant Pearson coefficient).

\subsection{Summarizing spatial patterns of urban diversification}

Principal Component Analysis (PCA) extracted 4 relevant components (eigenvalue > 1) explaining $72.9 \%$ of total matrix variance. A PCA biplot illustrates the joint distribution of urban dimensions and municipalities in the Athens' metropolitan regions based on component loadings and scores on the selected components (SM.Figure 2). Component 1 (34.3\%) identifies a traditional urban gradient opposing spatial patterns of diversification in building materials (negative loadings) to diversification in land-use, soil sealing profile, building's vertical profile, composition of nonnative population by citizenship and distribution of personal income (positive loadings). The highest scores on component 1 indicates urban municipalities belonging to the Greater Athens' area, while the lowest scores characterized the remaining municipalities of the study area (Figure 1). Component $2(17.2 \%)$ illustrates a gradient distinguishing municipalities with high and low diversification in economic base, building age, educational levels and composition of working classes. Apart from few exceptions, municipalities north, east and south of Athens received medium-high scores, while municipalities west of Athens had medium-low scores. Taken together, component 2 represents a gradient distinguishing wealth districts from economicallydisadvantaged areas, resembling the traditional east-to-west social differentiation in Athens. Component $3(13.0 \%)$ identifies a gradient discriminating the spatial patterns of three socio- 
1 demographic dimensions (positive loadings: educational levels; negative loadings: working class composition and population age structure). Western Attica and specific districts of eastern Attica (experiencing dispersed urban expansion in the last decades) received the highest scores on this component. Component $4(8.4 \%)$ was mainly associated with diversification in vertical profile of buildings, with the highest scores associated to central municipalities in the Greater Athens area.

\subsection{Cluster analysis}

A hierarchical clustering of municipalities based on the Pielou's J index of diversification in 12 analysis' dimensions identifies two groups of municipalities: urban districts and peri-urban/rural areas (Figure 2). Cluster analysis (SM.Figure 3) also grouped morphological attributes (e.g. landuse, soil sealing profile, vertical profile of buildings, settlement characteristics) and socioeconomic functions (e.g. working class composition, population age structure, distribution of personal incomes) on the base of similarities in their spatial distribution over the study area.

\subsection{Local-scale diversification in urban dimensions and metropolitan gradients}

Significant pair-wise Spearman correlation coefficients between each dimension of urban diversification and selected territorial attributes are reported in Table 4. Eight dimensions (all morphological attributes and two socioeconomic functions) were significantly correlated with variables oriented along the urban gradient (population density or distance from the most relevant urban nodes in the Athens' region) or indirectly associated with them (municipal area). Diversification in working class composition decreased with the distance from Markopoulo Messoghias, a central place in Messoghia district. Diversification in local-scale economic base, education levels and population age structure were not correlated with any contextual variable, indicating a spatial structure more influenced by place-specific factors than regional gradients.

\section{Discussion}

Considering the ties between settlement morphology and socioeconomic attributes, metropolitan complexity depends on a strong diversification in urban functions (Van Oort et al., 2015). In these regards, our study introduced a multivariate concept of metropolitan complexity based on a multi- 
1 domain analysis of indicators assessing morphological and socioeconomic dimensions of urban

2 diversity. To verify the spatial coherency of diversified urban functions, a multi-criteria spatial

3 analysis was adopted controlling for place-specific background variables. Indicators' systems have

4 been often proposed as more reliable and stable tools compared to individual variables (Salvati et

5 al., 2016a). Since relevant and updated variables characterizing metropolitan continuums or

6 discriminating urban from rural districts are still limited at both continental and country scale (e.g.

7 Hahs and McDonnell, 2006; Hoyler et al., 2008; Youn et al., 2016), our study proposes spatial

8 diversification in urban functions as a new indicator of metropolitan complexity. This approach may contribute to multi-domain information systems classifying urban, suburban and rural typologies of settlements in various regional contexts (Comer and Greene, 2015; Venerandi et al., 2017; Zambon et al., 2017).

Results of our study have identified urban and rural municipalities in Athens respectively as the most and less diversified, with suburban areas ranking in-between. Empirical results also demonstrate that local-scale morphological diversity is correlated with socioeconomic diversity in a non-linear manner. A correlation analysis with external variables oriented along the urban gradient indicates a different metropolitan structure characterizing each dimension of urban complexity. Territorial disparities result from the interaction among mixed social classes and selfgoverning micro-entities living in fragmented and heterogeneous 'island' settlements, outlining class segregation and economic inequalities at the spatial scale of neighbourhoods (Maloutas, 1993). In these regards, patterns of urban diversification in Athens reflect a divided spatial model, making this city a thought-provoking case with functional and morphological traits that exemplifies recent expansion of Mediterranean cities (Maloutas, 2007; Souliotis, 2013; Di Feliciantonio and Salvati, 2015).

Under the assumption that interplays between forms and functions contribute to urban diversification, the present study sheds light on present (and, possibly, future) challenges concerning the interplay between form and functions in southern Europe (Carlucci et al., 2017). For instance, economic polarization and social segregation should be considered and managed together, distinguishing local-scale heterogeneity from more general patterns characterizing regional-scale configurations (Rontos et al., 2016). In these regards, employment diversification has traditionally benefited from local economies (O’Donoghue, 1999; Ejermo, 2005; Ellerman, 2005). Employment has shifted from agriculture and industry towards a service- and information-based economy, defining new spatial patterns of specialization and diversification associated with inter- 
1 sectoral employment changes (O'Donoghue and Townshend, 2005). Measuring the intrinsic

2 diversity of economic activities in cities sheds more light on local development patterns and 3 suggests optimal (or suboptimal) structures that promote economic expansion and more 4 integrated urban systems (Youn et al., 2016).

5 A better understanding of socioeconomic transformations contributing to urban diversification 6 requires (i) a more integrated analysis of major challenges including social disparities, demographic transformations, economic resilience and environmental change (Duranton and Puga 2000; Desrochers, 2001; Wood and Dovey, 2015; Youn et al., 2016) and (ii) suitable planning measures recognizing that local-scale spatial structures may impact economic growth, social equity and sustainable urban development (Beriatos and Gospodini, 2004; Colantoni et al., 2016; Pili al., 2017). New lifestyle, gentrification, economic polarization and industrial proliferation are some of the main themes that regional policies and spatial planning should take care of (Smets and Salman, 2008), considering that housing quality, residential satisfaction, neighbourhood-based social interactions and residential attitudes towards social mix are negatively influenced by uncertain policy goals at the pertinent spatial level (Kleinhans, 2004).

Although diversification patterns are increasingly heterogeneous and difficult to dealt with integrally (e.g. Hirt, 2016), links among urban form and functions can be managed adapting land zoning targets to newly emerging metropolitan functions (Talen 2006; Vandermotten et al., 2008; Hirt 2012). While national policies targeting urban development have sometimes encouraged social mix and housing diversification in neighbourhoods under renewal and/or regeneration (O'Donoghue, 1999), a contemporary theory of difference should reinforce traditional interpretations of urban diversity and their connection with different political and economic contexts (Desrochers, 2001; Duranton and Puga, 2001; Ellerman, 2005;Kleinhans, 2004; Tochterman, 2012). Planning strategies adapting to an increased diversification of urban functions and measures promoting local development and metropolitan identity are particularly suitable for managing urban complexity.

\section{Conclusions}

Diversification in urban functions may effectively reflects a new metropolitan geography better than more classical indicators of urban growth. In this regard, evenness indexes allowed discrimination of metropolitan contexts with different settlement forms and socioeconomic 
1 dynamics, contributing to classify urban, suburban and rural areas. A multivariate spatial analysis based on diversification indexes proved to be a reliable tool to investigate local-scale urban complexity, identifying the latent relationship between the constituent dimensions, and may integrate decision support systems for diachronic analysis of urban expansion. The increasing impact of recent urbanization processes on metropolitan regions definitely requires a comparative and diachronic evaluation of the intimate relationship between land-use heterogeneity and socioeconomic diversification, considering together results from spatial statistics, mapping, inferential techniques and multivariate models. Innovative approaches that investigate linear and non-linear relationships among urban dimensions should be refined with analysis aimed at providing planners, policy-makers, economic actors and other stakeholders with an advanced knowledge to manage the increasing complexity of contemporary cities.

\section{Reference}

Batty, M., Longley, P.A. (1994). Fractal cities: a geometry of form and function. Academic press, London.

Beriatos, E., Gospodini, A. (2004). Glocalising urban landscapes: Athens and the 2004 Olympics Cities 21, 187-202.

Burger, M., Meijers, E. (2012). Form follows function? Linking morphological and functional polycentricity. Urban Studies 49(5), 1127-1149.

Cabral, P., Augusto, G., Tewolde, M., Araya, Y. (2013). Entropy in Urban Systems. Entropy 15(12), 5223-5236.

Carlucci, M., Grigoriadis, E., Rontos, K., Salvati, L. (2017). Revisiting a Hegemonic Concept: Longterm 'Mediterranean Urbanization' in Between City Re-polarization and Metropolitan Decline. Applied Spatial Analysis and Policy 10(3), 347-362.

Colantoni, A., Grigoriadis, E., Sateriano, A., Venanzoni, G., Salvati, L. (2016). Cities as selective land predators? A lesson on urban growth, deregulated planning and sprawl containment. Science of the Total Environment 545-546, 329339.

Colantoni, A., Mavrakis, A., Sorgi, T., Salvati, L. (2015). Towards a 'polycentric' landscape? Reconnecting fragments into an integrated network of coastal forests in Rome. Rendiconti Lincei 26, 615624 
1 Comer, D., Greene, J. (2015). The development and application of a land-use diversity index for Oklahoma City, OK. Applied Geography 60, 46-57.

Cuadrado-Ciuraneta, S., Durà-Guimerà, A., Salvati, L. (2017). Not only tourism: unravelling suburbanization, second-home expansion and "rural" sprawl in Catalonia, Spain 2017. Urban Geography 38(1), 66-89.

Dahly, D.L., Adair, L.S. (2007). Quantifying the urban environment: A scale measure of urbanicity outperforms the urban-rural dichotomy. Social Science \& Medicine 64(7), 1407-1419.

De Rosa, S., Salvati, L. (2016). Beyond a 'side street story'? Naples from spontaneous centrality to entropic polycentricism, towards a 'crisis city'. Cities 51, 74-83.

Desrochers, P. (2001). Local Diversity, Human Creativity and Technological Innovation. Growth and Change 32, 369-394.

Di Feliciantonio, C., Salvati, L. (2015). 'Southern' Alternatives of Urban Diffusion: Investigating Settlement Characteristics and Socio-Economic Patterns in Three Mediterranean Regions. Tijdschrift voor Economische en Sociale Geografie 106(4), 453470

Duranton, G., Puga, D. (2000). Diversity and Specialisation in Cities: Why, Where and When Does it Matter? Urban Studies 37, 533-555.

Duvernoy, I., Zambon, I., Sateriano, A., Salvati, L. (2018). Pictures from the other side of the fringe: Urban growth and peri-urban agriculture in a post-industrial city (Toulouse, France). Journal of Rural Studies 57, 2535.

Ejermo, O. (2005). Technological diversity and Jacobs' externality hypothesis revisited. Growth and Change 36(2), 167-195.

Ellerman, D. (2005). How Do We Grow?: Jane Jacobs on Diversification and Specialization. Challenge 48(3), 50-83.

European Environment Agency (2011). Mapping guide for a European urban atlas. EEA: Copenhagen (Version 1.1).

Frenken, K., Van Oort, F., Verburg, T. (2007). Related variety, unrelated variety and regional economic growth, Regional Studies 41, 685-697.

Grekousis, G., Manetos, P., Photis, Y.N. (2013). Modeling urban evolution using neural networks, fuzzy logic and GIS: The case of the Athens metropolitan area. Cities 30, 193-203.

Hahs, A.K., McDonnell, M.J. (2006). Selecting independent measures to quantify Melbourne's urban-rural gradient. Landscape and Urban Planning 78(4), 435-448. 
Hirt, S. (2012). Mixed use by default: how the Europeans (don't) zone. Journal of Planning Literature 27(4), 375-393.

Hirt, S.A. (2016). Rooting out mixed use: Revisiting the original rationales. Land Use Policy 50, 134147.

Hoyler, M., Kloosterman, R.C., Sokol, M. (2008). Polycentric puzzles: emerging megacity regions seen through the lens of advanced producer services. Regional Studies 42, 1055-1064.

Kazemzadeh-Zow, A., Zanganeh Shahraki, S., Salvati, L., Samani, N.N. (2017). A spatial zoning approach to calibrate and validate urban growth models. International Journal of Geographical Information Science 31(4), 763782.

Kleinhans, R. (2004). Social implications of housing diversification in urban renewal: A review of recent literature. Journal of Housing and the Built Environment, 19(4), 367-390.

Kroll, F., Kabisch, N. (2012). The Relation of Diverging Urban Growth Processes and Demographic Change along an Urban-Rural Gradient. Population, Space and Place 18(3), 260-276.

Malheiros, J. (2002). Ethni-cities: residential patterns in the northern European and Mediterranean metropolises - implications for policy design. International Journal of Population Geography $8(2), 107-134$.

Maloutas, T. (1993). Social segregation in Athens. Antipode 25(3), 223-239.

Maloutas, T. (2007). Socioeconomic Classification Models and Contextual Difference: The European Socioeconomic Classes (ESeC) from a South European Angle. South European Society and Politics 12(4), 443-460.

Markusen, A., Schrock, G. (2006). The distinctive city: divergent patterns in growth, hierarchy and specialisation. Urban Studies 43(8), 1301-1323.

Meijers, E., Burger, M. (2010). Spatial structure and productivity in US metropolitan areas, Environment and Planning A 42, 1383-1402.

Munafò, M., Salvati, L., Zitti, M. (2013). Estimating soil sealing rate at national level - Italy as a case study. Ecological Indicators 26, 137-140.

Mustafa, A., Van Rompaey, A., Cools, M., Saadi, I., Teller, J. (2018). Addressing the determinants of built-up expansion and densification processes at the regional scale. Urban Studies 55(15), 32793298.

Pili, S., Grigoriadis, E., Carlucci, M., Clemente, M., Salvati, L. (2017). Towards sustainable growth? A multi-criteria assessment of (changing) urban forms. Ecological Indicators 76, 71-80. 
1 O'Donoghue, D. (1999). The relationship between diversification and growth: some evidence from the British urban system 1978 to 1991. International Journal of Urban and Regional Research 23(3), 549-566.

O'Donoghue, D., Townshend, I.J. (2005). Diversification, specialization, convergence and divergence of sectoral employment structures in the British urban system, 1991-2001. Regional Studies 39, 585-601.

Ottaviano, G.I., Peri, G. (2006). The economic value of cultural diversity: evidence from US cities. Journal of Economic Geography 6(1), 9-44.

Page, M., Parisel, C., Pumain, D., Sanders, L. (2001). Knowledge-based simulation of settlement systems. Computers, Environment and Urban Systems 25(2), 167-193.

Parr, J.B. (2008). Cities and regions: problems and potentials. Environment and Planning A 40, 3009-3026.

Parr, J.B. (2004). The polycentric urban region: a closer inspection. Regional Studies 38, 231-240.

Riguelle, F., Thomas, I., Verhetsel, A. (2007). Measuring urban polycentrism: a European case study and its implications. Journal of Economic Geography, 7(2), 193-215

Rontos, K., Grigoriadis, E., Sateriano, A., Syrmali, M., Vavouras, I., Salvati, L. (2016). Lost in protest, found in segregation: Divided cities in the light of the 2015 "O $\chi \imath$ ” referendum in Greece. City, Culture and Society, 7(3), 139-148.

Salvati, L. (2013). Monitoring high-quality soil consumption driven by urban pressure in a growing city (Rome, Italy). Cities 31, 349-356.

Salvati, L. (2014). Agro-forest landscape and the 'fringe' city: A multivariate assessment of land-use changes in a sprawling region and implications for planning. Science of the Total Environment $490,715-723$.

Salvati, L., Sateriano, A., Saradakou, E., Grigoriadis, E. (2016a). 'Land-use mixité': Evaluating urban hierarchy and the urban-to-rural gradient with an evenness-based approach. Ecological Indicators 70, 35-42.

Salvati, L., Venanzoni, G., Serra, P., Carlucci, M. (2016b). Scattered or polycentric? Untangling urban growth in three southern European metropolitan regions through exploratory spatial data analysis. Annals of Regional Science 57(1), 1-29.

Serra, P., Vera, A., Tulla, F., Salvati, L. (2014). Beyond urban-rural dichotomy: exploring socioeconomic and land-use processes of change in Spain (1991-2011). Applied Geography 55, 71-81. 
Smets, P., Salman, T. (2008). Countering urban segregation: Theoretical and policy innovations from around the globe. Urban Studies 45(7), 1307-1332.

Souliotis, N. (2013). Cultural economy, sovereign debt crisis and the importance of local contexts: The case of Athens. Cities 33, 61-68.

Stanley, B. (2012). An historical perspective on the viability of urban diversity: lessons from sociospatial identity construction in nineteenth-century Algiers and Cape Town. Journal of Urbanism $5(1), 67-86$.

Talen, E. (2006). Design that enables diver-sity: The complications of a planning ideal. Journal of Planning Literature 20(3), 233-249.

Thomas, I., Frankhauser, P., Badariotti, D. (2012). Comparing the fractality of European urban neighbourhoods: do national contexts matter? Journal of Geographical Systems, 14(2), 189-208.

Tochterman, B. (2012). Theorizing neoliberal urban development: A genealogy from Richard Florida to Jane Jacobs. Radical History Review (112), 65-87.

Torrens, P.M. (2008). A toolkit for measuring sprawl. Applied Spatial Analysis and Policy 1, 5-36.

Turok, I., Bailey, N. (2004). The theory of polynuclear urban regions and its application to central Scotland. European Planning Studies 12, 371-389.

Tsai, Y-H (2005). Quantifying Urban Form: Compactness versus 'Sprawl'. Urban Studies 42(1), 141161.

Vandermotten, C., Halbert, L., Roelandts, M., Cornut, P. (2008). European planning and the polycentric consensus: wishful thinking? Regional Studies 42, 1205-1217.

Van Oort, F., de Geus, S., Dogaru, T. (2015). Related variety and regional economic growth in a cross-section of European urban regions. European Planning Studies 23(6), 1110-1127.

Vasanen, A. (2012). Functional polycentricity: examining metropolitan spatial structure through the connectivity of urban sub-centres. Urban Studies 49(16), 3627-3644.

Venerandi, A., Zanella, M., Romice, O., Dibble, J., Porta, S. (2017). Form and urban change - An urban morphometric study of five gentrified neighbourhoods in London. Environment and Planning B: Urban Analytics and City Science 44(6), 1056-1076.

Wood, S., Dovey, K. (2015). Creative Multiplicities: Urban Morphologies of Creative Clustering. Journal of Urban Design 20(1), 52-74.

Youn, H., Bettencourt, L.M., Lobo, J., Strumsky, D., Samaniego, H., West, G.B. (2016). Scaling and universality in urban economic diversification. Journal of The Royal Society Interface 13(114), 20150937. 
1 Zambon, I., Serra, P., Grigoriadis, E., Carlucci, M., Salvati, L. (2017). Emerging urban centrality: An

2 entropy-based indicator of polycentric development and economic growth. Land Use Policy 68, $3 \quad 365-371$.

4 Zambon, I., Benedetti, A., Ferrara, C., Salvati, L. (2018). Soil Matters? A Multivariate Analysis of 5 Socioeconomic Constraints to Urban Expansion in Mediterranean Europe. Ecological Economics $6 \quad 146,173-183$.

7 Zitti, M., Ferrara, C., Perini, L., Carlucci, M., Salvati, L. (2015). Long-term urban growth and land 8 use efficiency in Southern Europe: Implications for sustainable land management. Sustainability $9 \quad 7(3), 33593385$. 